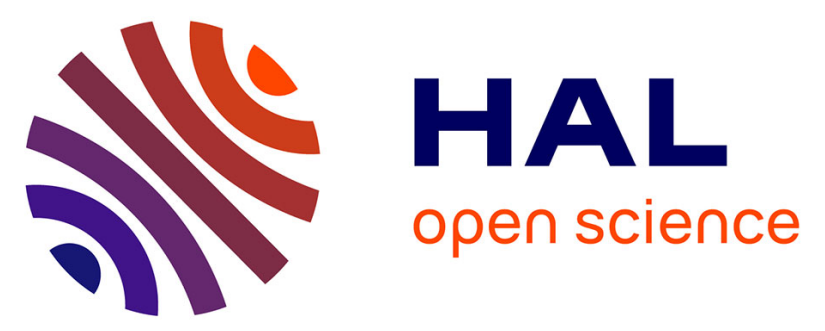

\title{
Juvenile, but not adult exposure to high-fat diet impairs relational memory and hippocampal neurogenesis in mice
}

Chloé Boitard, Nicole Etchamendy, Julie Sauvant, Agnès Aubert, Sophie Tronel, Aline Marighetto, Sophie Layé, Guillaume Ferreira

\section{To cite this version:}

Chloé Boitard, Nicole Etchamendy, Julie Sauvant, Agnès Aubert, Sophie Tronel, et al.. Juvenile, but not adult exposure to high-fat diet impairs relational memory and hippocampal neurogenesis in mice. Hippocampus, 2012, 22 (11), pp.2095-2100. 10.1002/hipo.22032 . hal-02326398

\section{HAL Id: hal-02326398 \\ https://hal.science/hal-02326398}

Submitted on 29 May 2020

HAL is a multi-disciplinary open access archive for the deposit and dissemination of scientific research documents, whether they are published or not. The documents may come from teaching and research institutions in France or abroad, or from public or private research centers.
L'archive ouverte pluridisciplinaire HAL, est destinée au dépôt et à la diffusion de documents scientifiques de niveau recherche, publiés ou non, émanant des établissements d'enseignement et de recherche français ou étrangers, des laboratoires publics ou privés. 


\title{
RAPID COMMUNICATION
}

\section{Juvenile, but Not Adult Exposure to High-Fat Diet Impairs Relational Memory and Hippocampal Neurogenesis in Mice}

\author{
Chloe Boitard, ${ }^{1,2}$ Nicole Etchamendy, ${ }^{1,2}$ Julie Sauvant, ${ }^{1,2}$ Agnes Aubert, ${ }^{1,2}$ Sophie Tronel, ${ }^{3,4}$ \\ Aline Marighetto, ${ }^{3,4}$ Sophie Layé, ${ }^{1,2}$ and Guillaume Ferreira ${ }^{1,2 *}$
}

\begin{abstract}
Increased consumption of high-fat diet (HFD) leads to obesity and adverse neurocognitive outcomes. Childhood and adolescence are important periods of brain maturation shaping cognitive function. These periods could consequently be particularly sensitive to the detrimental effects of HFD intake. In mice, juvenile and adulthood consumption of HFD induce similar morphometric and metabolic changes. However, only juvenile exposure to HFD abolishes relational memory flexibility, assessed after initial radial-maze concurrent spatial discrimination learning, and decreases neurogenesis. Our results identify a critical period of development covering adolescence with higher sensitivity to HFD-induced hippocampal dysfunction at both behavioral and cellular levels. $\odot 2012$ Wiley Periodicals, Inc.
\end{abstract}

KEY WORDS: adolescence; overweight; spatial learning; memory; neurogenesis

Overweight and obesity, one of the most serious public health challenges of the 21st century, are mainly due to overconsumption of energy-dense food, particularly high-fat diet (HFD). In addition to adverse health outcomes causing disability and premature death, they are associated with cognitive disturbances. Declarative memory, which critically depends on the hippocampus, is particularly affected in overweight and obese adults (Nilsson and Nilsson, 2009). Overweight children and adolescents also show cognitive impairments (Cserjesi et al., 2007; Li et al., 2008). This can be particularly problematic as childhood and adolescence are crucial periods for the maturation of some brain structures, like the hippocampus, necessary for shaping cognitive function (Spear, 2000). Importantly, these early life periods are very sensitive to environmental challenges, like food intake (Andersen, 2003), and could therefore be particularly susceptible to the adverse effect of HFD consumption.

In animal models, western diet consumption induces overweight and can affect learning and memory functions, especially those depending on the hippocampus (for review, see Kanoski and Davidson, 2011). In addition to this behavioral effect, HFD consumption can also decrease hippocampal neurogenesis, a form of plasticity based on persistent formation of

\footnotetext{
${ }^{1}$ INRA, Nutrition et Neurobiologie Intégrée, UMR1286, F-33000 Bordeaux, France; ${ }^{2}$ Université de Bordeaux, Nutrition et Neurobiologie Intégrée, UMR 1286, F-33000 Bordeaux, France; ${ }^{3}$ INSERM, Neurocentre Magendie, U862, F-33000 Bordeaux, France; ${ }^{4}$ Université de Bordeaux, Neurocentre Magendie, U862, F-33000 Bordeaux, France Grant sponsors: AXA, Emergence de Jeune Equipe INRA

*Correspondence to: Guillaume Ferreira, Nutrition and Integrative Neurobiology (NutriNeuro), INRA 1286 Université de Bordeaux, Bâtiment UFR Pharmacie, 146 rue Léo Saignat, 33076 Bordeaux, France.

E-mail: guillaume.ferreira@bordeaux.inra.fr

Accepted for publication 4 April 2012

DOI 10.1002/hipo.22032

Published online 17 May 2012 in Wiley Online Library

(wileyonlinelibrary.com).
}

adult-born neurons in the dentate gyrus (Lindqvist et al., 2006; Hwang et al., 2008; Park et al., 2010). This is of particular importance as hippocampal neurogenesis participates in hippocampal-dependent memory, particularly flexible memory expression (Koehl and Abrous, 2011). However, the existence of a particular early life period of vulnerability to the detrimental effects of HFD on cellular and behavioral function of the hippocampus remains unexplored. Thus, we compare the effects of the same duration of HFD consumption in mice, starting at weaning (covering adolescence) or at adulthood, on hippocampal neurogenesis and behavioral function. We first assessed radial-maze concurrent spatial discrimination learning of three pairs of arms (Marighetto et al., 1999; Touzani et al., 2003). Second, memory flexibility was evaluated by presenting a novel recombined pair of arms. Memory flexibility relying on a relational representation of previously acquired separate experiences (i.e. initial learning; Marighetto et al., 1999) is mediated by the hippocampus in mice (Touzani et al., 2003; see also Etchamendy et al., 2003; Mingaud et al., 2007) and represents an interesting model of human declarative memory (see Etchamendy et al., 2012).

Naïve C57BL6/J male mice (Janvier, Le Genest St. Isle, France) aged either 3 weeks old ( $n=27$, weaning groups) or 12 weeks old ( $n=26$, adult groups) when placed on either standard laboratory chow, control diet (CD) offering $2.9 \mathrm{kcal} / \mathrm{g}$ [consisting of $60 \%$ carbohydrate, mostly from starch, and 3\% fat (A04 SAFE, Augy, France)], or HFD offering $4.7 \mathrm{kcal} / \mathrm{g}$ [consisting of $24 \%(45 \% \mathrm{kcal})$ fat, mostly saturated fat from lard, and $41 \%$ (35\% kcal) carbohydrate, with $20 \%(17.5 \% \mathrm{kcal})$ from sucrose (D12451, Research Diets, New Brunswick, NJ)]. Mice were housed in groups of three per cage with ad libitum access to food and water until 8 days prior to behavioral assessment, where they were housed individually.

Relational memory flexibility was assessed after 11 weeks on HFD or CD using a two-stage radial-maze concurrent spatial discrimination learning. The apparatus was a fully automated, elevated, eight-arm radial maze, and the procedure used was fully described elsewhere (Marighetto et al., 1999). Briefly, the amount of 

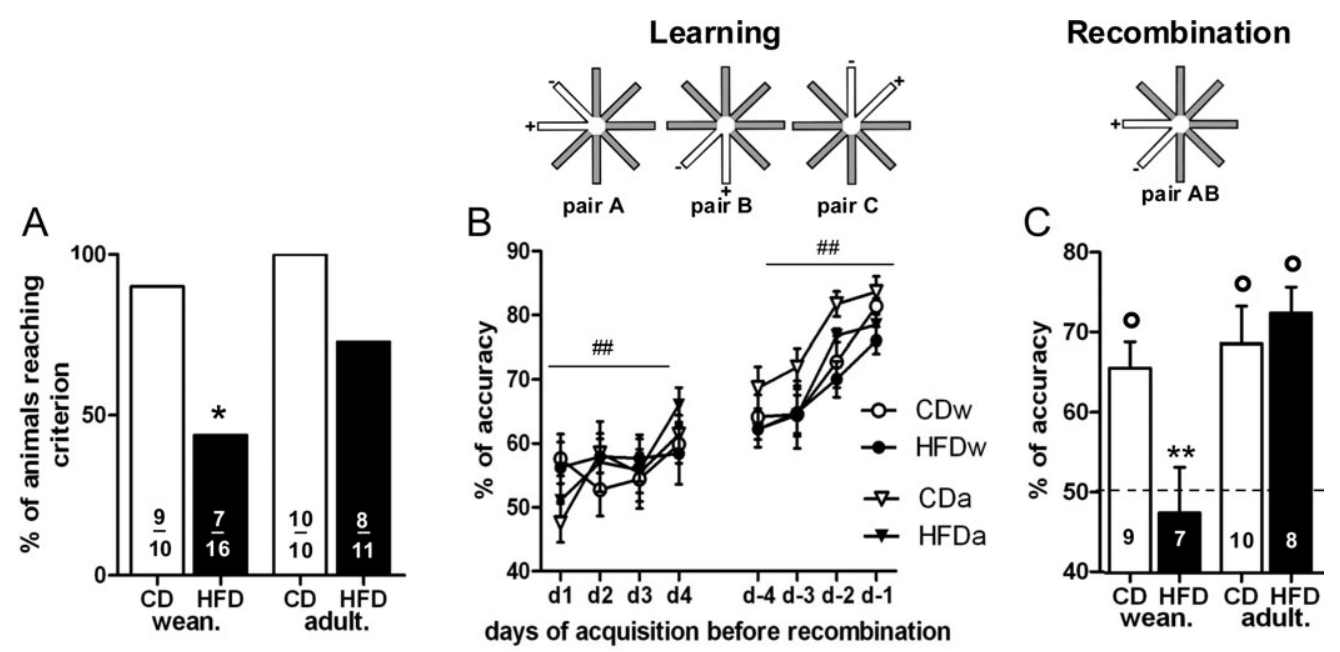

FIGURE 1. Effect of HFD consumption since weaning or adulthood on spatial learning and relational memory. A: Percentage of mice reaching the criterion of $75 \%$ accuracy in Pairs $A$ and B. B: Schematic representation of the three pairs used during learning (the baited arm is represented by + and the not baited arm by - for each pair; top) with the percentage of accuracy (mean \pm SEM) in the three pairs over the first four and the last

food given was first controlled to maintain the original bodyweight down to $85 \%$ for each mouse. The animals were then habituated to go eating in each arm of the apparatus over 2 days. Six mice did not habituate and were thus excluded. Each mouse was then randomly assigned three adjacent pairs of arms (Pairs A, B, and C; Fig. 1B), each made of a positive (baited) and a negative (not baited) arm. Each daily session consisted of 20 consecutive trials comprising presentations of each pair according to a pseudo-random sequence. In each trial, the animal was confronted with access to both arms of a pair. A choice was considered to be made when the subject reached the food well located at the extremity of the arm. All mice reached the food well located at the extremity of the arm (and ate pellet in baited arm) for each of the 20 daily trials within each of the learning days, with no differences in the time that animals took to reach the reward when comparing the chow-fed and HFDfed groups. The end of the trial consisted in the animal returning to the central platform, where it was confined for $5 \mathrm{~s}$ (intertrial interval) before the beginning of the next trial. A performance criterion was defined as the mouse reaching an overall choice accuracy of $70 \%$ over two successive days with at least $75 \%$ accuracy for Pairs A and B. Once the mouse reached this criterion, it was given the recombination task. If the mouse failed to reach the criterion within the 12 learning days allowed, it was excluded from further testing. During the recombination task, for two consecutive days, animals were confronted to 20 consecutive trials with presentations of recombined pair $A B$ (Fig. 1C), Pairs $\mathrm{C}$ and $\mathrm{N}$ according to a pseudo-random sequence. Recombined pair $A B$ consisted of the association of the positive arm from Pair A (or B) with the negative arm of Pair B (or A). Pair C was the familiar pair seen in the learning stage (positive control), and Pair $\mathrm{N}$ was a novel pair (negative control).

four learning sessions before achieving criterion (below). C: Schematic representation of a recombined pair $A B$ (top) with the percentage of accuracy (mean $\pm S E M$ ) in recombined pair (below). ${ }^{*} P<0.05$ when compared with the corresponding control groups; ${ }^{* *} P<0.01$ when compared with the other groups; ${ }^{\circ} \boldsymbol{P}<0.05$ different from chance level; ${ }^{\# \#} P<0.01$ increased performance over session.

Neurogenesis in the hippocampus was evaluated by determining the number of immature neurons in the dentate gyrus characterized by the endogenous marker doublecortin (DCX), a cytoplasmic protein expressed transiently in newborn neurons only (Brown et al., 2003). To avoid a potential effect of spatial learning on neurogenesis (Tronel et al., 2010), DCX immunolabeling was performed 4 weeks after the end of spatial-relational memory assessment. Briefly, $40-\mu \mathrm{m}$ sections (spaced $240 \mu \mathrm{m}$ apart) were incubated with diluted rabbit anti-DCX antibody (1/800; Abcam, Cambridge, UK) followed by biotinylated secondary antibody and streptavidin peroxidase complex, which was visualized by diaminobenzidine-nickel staining. The number of DCX positive cells was counted on four sections representing the same four levels of the dentate gyrus for all animals.

The same duration of HFD intake, starting at weaning or at adulthood, induced similar overweight and metabolic changes (Table 1). During concurrent spatial discrimination learning, a signicantly lower proportion of animals reached criterion in the HFD-fed group since weaning (HFDw) when compared with their control group (CDw, Fisher's exact test, 7/16 vs. 9/10, $P=$ 0.037), whereas the HFD-fed and CD groups since adulthood did not differ (HFDa and CDa, 8/11 vs. 10/10, respectively, $P$ $=0.21$; Fig. $1 \mathrm{~A}$ ). However, the effect of the diet on the proportion of mice reaching criterion did not significantly vary by age (age $\times$ diet interaction; GLM for binary data, $P=0.47$ ). Only the subjects reaching criterion during simultaneous discrimination were kept for further analysis. The percentage of correct choice in the three pairs increased in all groups over the first four $\left(F_{3,90}=3.7, P=0.015\right)$ and the last four learning sessions $\left(F_{3,90}=29.1, P<0.001\right)$ and was not different between groups $\left(F_{3,90}<1\right.$ for sessions $\times$ age $\times$ diet interaction; Fig. 1B). When only Pairs A and B were taken into account on the last learning day before recombination, all animals achieved at least 
TABLE 1.

Body weight, food intake and metabolic parameters in HFD-fed mice

\begin{tabular}{|c|c|c|c|c|}
\hline & \multicolumn{2}{|c|}{ Weaning } & \multicolumn{2}{|c|}{ Adulthood } \\
\hline & $\mathrm{CD}$ & HFD & $\mathrm{CD}$ & HFD \\
\hline Body weight before behavior* & $28.0 \pm 0.3$ & $29.1 \pm 0.4^{\dagger}$ & $30.2 \pm 0.8$ & $33.0 \pm 1.2^{\dagger}$ \\
\hline Final body weight ${ }^{*}$ & $28.9 \pm 0.4$ & $34.4 \pm 0.7^{\ddagger}$ & $31.5 \pm 0.8$ & $38.8 \pm 1.5$ \\
\hline Food intake (g/day) & $4.6 \pm 0.2$ & $3.0 \pm 0.7^{*}$ & $4.3 \pm 0.5$ & $3.4 \pm 0.5$ \\
\hline Insulin $(\mathrm{ng} / \mathrm{ml})$ & $1.2 \pm 0.2$ & $3.2 \pm 0.4$ & $1.1 \pm 0.2$ & $2.9 \pm 0.5$ \\
\hline Corticosterone (ng/ml) & $24.6 \pm 8.2$ & $56.9 \pm 6.9^{*}$ & $31.8 \pm 4.3$ & $47.7 \pm 5.6$ \\
\hline Cholesterol $(\mathrm{mg} / \mathrm{dl})^{*}$ & $81.6 \pm 5.4$ & $145.3 \pm 5.0^{t}$ & $95.1 \pm 3.2$ & $166.3 \pm 6.6$ \\
\hline Triglycerides (mg/dl) & $47.6 \pm 4.5$ & $52.5 \pm 3.7$ & $46.5 \pm 2.9$ & $56.4 \pm 8.6$ \\
\hline Glucose $(\mathrm{mg} / \mathrm{dl})^{*}$ & $130.3 \pm 4.7$ & $133.7 \pm 2.8$ & $123.1 \pm 3.0$ & $121.7 \pm 5.6$ \\
\hline
\end{tabular}

The 3- or 12-week-old-mice were fed CD or HFD for 17 weeks. The HFD-fed groups weighted 4-10\% more than their corresponding CD-fed group just before the beginning of behavioral assessment and 19-23\% more at the time of sacrifice. Food and energy intake was measured during 9 weeks prior to behavioral assessment. HFD intake starting at weaning and at adulthood similarly increased basal plasmatic levels of cholesterol, insulin, leptin, and corticosterone and did not modify the basal level of triglycerides and glucose. Blood glucose level was assessed in 24-h food-deprived animals by tail nick using Accu-Check ${ }^{\mathbb{R}}$ devices (Roche Diagnostics, France) after 15 weeks on diets. The other metabolic parameters were measured in plasma obtained from blood collected at sacrifice using specific kits or bioplex (Biomérieux, France; Millipore, Billerica) after 17 weeks on diets. Total corticosterone was measured by an in-house radioimmunoassay (Richard et al., 2010).

${ }^{*} \mathrm{P}<0.05$ : age difference; ${ }^{\dagger} \mathrm{P}<0.06$ and ${ }^{\dagger} \mathrm{P}<0.05$ when compared with corresponding control group.

$75 \%$ of accuracy, and the performance was not different between groups $\left(F_{1,30}<1\right.$ for age $\times$ diet interaction).

During assessment of relational memory flexibility, the HFDw group was severely impaired. Performance of $\mathrm{CDw}$, $\mathrm{CDa}$, and HFDa groups were well above chance in the recombined pair $\mathrm{AB}$ (one-sample $t$-test, $t_{8}=4.9, t_{9}=4.0$, and $t_{7}=$ 7.1, respectively; $P<0.003$ ), whereas performance of HFDw group was not different from chance level $\left(t_{6}<1, P=0.6\right.$; Fig. 1C) and was significantly lower than the other groups $\left(F_{1,30}=6.5, P=0.016\right.$ for age $\times$ diet interaction; post hoc $P$ $<0.007$ between HFDw group and the other three groups, $P$ $>0.1$ for all other comparisons). In the previously learned pair (Pair C), all the groups performed above chance level (one-sample $t$-test, $P=0.07$ for HFDw group and $P<0.002$ for the other groups), and there was no difference between groups $\left(F_{1,30}<1\right.$ for age $\times$ diet interaction; data not shown). In the other control condition consisting in exposure to a novel pair (Pair N), all the groups performed at chance level (one-sample $t$-test, $P>0.05$ for all groups), and there was no difference between groups $\left(F_{1,30}=1.3, P>0.1\right.$ for age $\times$ diet interaction; data not shown).

Exposure to the HFD resulted in a significant decrease in the number of DCX-positive cells when intake started at weaning (23\% reduction), but not when it started at adulthood (Fig. 2). This was reflected by a significant age by diet interaction $\left(F_{1,39}=4.5, P=0.039\right.$, post hoc, $P=0.0014$ between HFDw and CDw groups, $P=0.9$ between $\mathrm{CDa}$ and $\mathrm{HFa}$ groups). Moreover, $\mathrm{CDw}$ group (aged 20 weeks old at the time
A

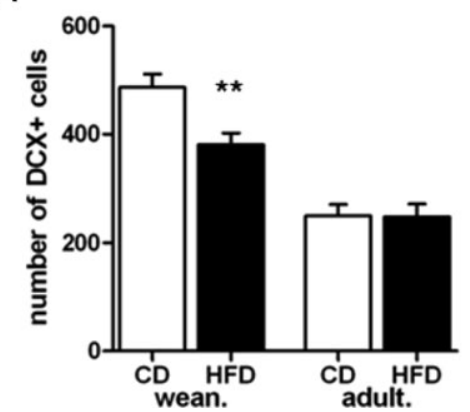

B $\quad C D$

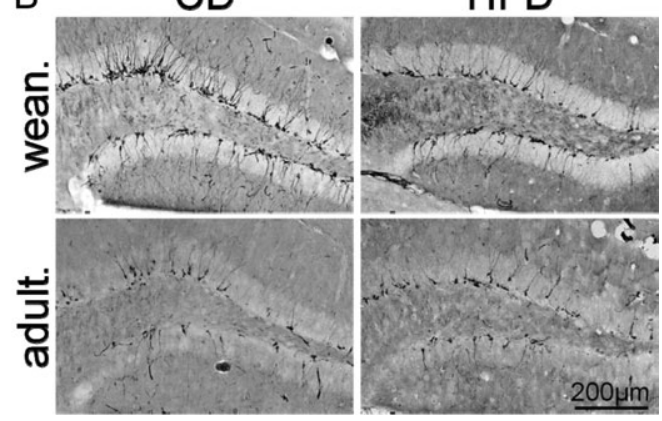

FIGURE 2. Effect of HFD consumption since weaning or adulthood on hippocampal neurogenesis. A: Number of DCX-positive cells $(\mathrm{DCX}+$, mean $\pm \mathrm{SEM})$ in the dentate gyrus of the hippocampus. B: Representative DCX staining in the dentate gyrus of the hippocampus for each group. ${ }^{* *} P<0.01$ when compared with corresponding control group. 
of sacrifice) had twofold higher number of new neurons than CDa group (aged 29 weeks old at the time of sacrifice; $P<$ 0.05), as previously reported (He and Crews, 2007).

Our results show that early life consumption of HFD, from weaning to adulthood, results in loss of relational memory flexibility as well as decreased hippocampal neurogenesis. Yet, the same duration of HFD consumption confined at adulthood does not yield such adverse consequences. Therefore, early life period appears to be particularly vulnerable to the detrimental effects of HFD consumption on hippocampal functioning at both behavioral and cellular levels.

HFD-fed mice that fail the relational memory show intact spatial discrimination learning although both relational memory and spatial learning depend on the hippocampus. However, it has been repeatedly observed that despite having mastered the initial spatial discrimination learning, performance of aged subjects (mice or humans) drops dramatically during the relational memory assessment (aged mice: Marighetto et al., 1999; Touzani et al., 2003; aged humans: Etchamendy et al., 2012). This suggests that relational memory is a higher challenging cognitive task than spatial discrimination learning, which is more amenable to reveal subtle hippocampal dysfunction induced for instance by juvenile exposure to HFD.

The adolescence period for rodents ranges, in a nonrestrictive way, from the time of weaning ( 3 weeks old) to 8 weeks old. This is followed by a transition period leading to adulthood that clearly begins after 12 postnatal weeks (Spear, 2000; Andersen, 2003). In our study, early life period of HFD consumption starting at weaning therefore encompasses adolescence, whereas HFD consumption starting when mice were 12 weeks old corresponds exclusively to adulthood challenge. Similar to our results, some studies have found deficits in hippocampal-dependent memory (assessed in radial-arm maze or Morris water maze) when HFD consumption starts at 3-4 weeks of age and lasts at least 2-3 months (Greenwood and Winocur, 2005; Valladolid-Acebes et al., 2011) but not when it starts after 8 weeks of age (Mielke et al., 2006; White et al., 2009; McNeilly et al., 2011). In contrast to these results, some studies report impairment in hippocampal-dependent memory after HFD consumption at adulthood. However, in comparison with our study, these studies used different behavioral paradigms (Morris water maze, Stone T-maze, and radial-maze test of working memory) and different diet-related parameters: a very long duration of HFD consumption (Farr et al., 2008), a very high percentage of HFD (Murray et al., 2009; Pistell et al., 2010), or a combination of HFD with high sugar (Molteni et al., 2002; Stranahan et al., 2008; Kanoski and Davidson, 2010). Combining these findings with ours, we may suggest that early life period is particularly sensitive to the deleterious effects of HFD on hippocampal memory and that adulthood is a less vulnerable period yet not immune to this adverse effect of HFD. Our results also corroborate recent behavioral findings showing that HFD consumption during adolescence impaired conditioned place preference (using HFD reward), whereas later HFD consumption had no effect (Privitera et al., 2011). However, the peripheral or central mecha- nisms linked to such vulnerability were not investigated in this study.

In parallel to the memory impairment seen only in mice fed with HFD during early life, we found specifically in these mice a decrease in the number of new neurons in the dentate gyrus. Previous studies indicate that HFD consumption is able to decrease the different steps of adult hippocampal neurogenesis, that is, proliferation of neural progenitor cells, neuronal differentiation, and newborn cells survival (Lindqvist et al., 2006; Hwang et al., 2008; Park et al., 2010), albeit a recent report did not find any effects of HFD consumption on cell proliferation in the dentate gyrus (Rivera et al., 2011). In this study, the HFD consumption started when rats were 12 weeks old, whereas younger animals ( $\sim 6$ weeks old) were used in the other studies. This corroborates our finding on the vulnerability of the early life period to the effect of HFD on hippocampal neurogenesis. Hippocampal neurogenesis and more specifically the integration of adult-born neurons into the hippocampal circuitry participate in learning and memory (Koehl and Abrous, 2011). In particular, disrupting neurogenesis impairs the ability to use previously learned information in a novel situation indicating that adult-born neurons are critical for flexible, inferential memory expression (Dupret et al., 2008). The task we used is based on relational memory flexibility, and performing this task induces activation of the different hippocampal subregions, including the dentate gyrus (Touzani et al., 2003). Therefore, the specific hippocampaldependent memory impairment seen in HFD-fed mice during early life seems related to the decrease in hippocampal neurogenesis.

What remains to be established is how hippocampal neurogenesis can be affected by HFD in early exposed mice. Neurogenesis can be lowered by high levels of the stress hormone corticosterone (Joels, 2007). As HFD-fed animals that show decreased neurogenesis also exhibit elevated corticosterone levels (Lindqvist et al., 2006), it was proposed that corticosterone mediates the deleterious effects of HFD intake on neurogenesis. In our conditions, early life and adult HFD consumption similarly increased circulating corticosterone, whereas only early life HFD consumption decreased hippocampal neurogenesis. Nevertheless, the hypothalamic-pituitary-adrenal axis (regulating corticosterone levels) matures during adolescence (Lupien et al., 2009), thereby providing opportunity for high levels of glucocorticoids to produce more drastic changes on hippocampal function in juvenile than in adult animals. Therefore, it would be interesting to more carefully evaluate the impact of HFD consumption during adolescence and adulthood on this axis. Brain inflammation also decreases hippocampal neurogenesis (Ekdahl et al., 2009), and HFD consumption was associated with brain inflammation and spatial memory impairment (Pistell et al., 2010). The causal link between increased hippocampal inflammation following HFD intake during adolescence and decreased neurogenesis and cognitive performance remains to be elucidated.

Previous studies suggest that metabolic changes due to HFD consumption could be responsible for cognitive impairment. 
For instance, hypertriglyceridemia induced by 10 months of HFD intake mediated cognitive deficit in CD-1 mice (Farr et al., 2008). In our study, HFD consumption for 4 months did not elevate triglycerides in either early or adult C57BL6/J mice (see also Hwang et al., 2010 for similar results in the same strain). The discrepancy between studies could therefore be due to the duration of HFD consumption and/or to the mouse strain. Higher levels of insulin, glucose, or cholesterol induced by HFD intake have also been linked to cognitive impairment (Greenwood and Winocur, 2005; Stranahan et al., 2008). However, we found similar changes in all these metabolic parameters in both early and adult HFD-exposed groups, whereas only early HFD-exposed mice were cognitively impaired. Early and adult HFD consumption also induced similar overweight and no deficit in food motivation during the task. Therefore, the cognitive impairment seen specifically in mice fed with HFD during early life seems not to be related to HFD-induced metabolic, morphometric, or motivational changes.

In conclusion, HFD exposure in early life (i.e., during adolescence) decreases hippocampal neurogenesis and abolishes relational memory flexibility. Importantly, the flexibility property of relational memory assessed here is a well-defined model of declarative memory (Marighetto et al., 1999; Etchamendy et al., 2012), which is particularly affected in obese humans (Nilsson and Nilsson, 2009). Therefore, it seems of a major concern to investigate how saturated fat diet consumption can have deeper cognitive impact when occurring during adolescence by targeting the maturing brain. A better understanding is needed to prevent the potentially strong cognitive impairment in the nowadays growing population of obese children and adolescents, which may lead to long-lasting cognitive disability.

\section{Acknowledgments}

The authors thank Anais Gaillard, Brenda Lopez-Bazan, Laurent Brayda-Bruno, and Jean-Christophe Delpech for technical assistance; Philippe Birac for taking care of the animals; and Dr. Hubert Vaudry (University of Rouen, France) for providing the anticorticosterone antibody. They also thank Maxime Hervé and Denis Poinsot (University of Rennes 1 France) for their help with statistical analyses, and Claire Dawson for the English revision of the final text.

\section{REFERENCES}

Andersen SL. 2003. Trajectories of brain development: Point of vulnerability or window of opportunity? Neurosci Biobehav Rev 27:318.

Brown JP, Couillard-Despres S, Cooper-Kuhn CM, Winkler J, Aigner L, Kuhn HG. 2003. Transient expression of doublecortin during adult neurogenesis. J Comp Neurol 467:1-10.

Cserjesi R, Molnar D, Luminet O, Lenard L. 2007. Is there any relationship between obesity and mental flexibility in children? Appetite 49:675-678.
Dupret D, Fabre A, Dobrossy MD, Panatier A, Rodriguez JJ, Lamarque S, Lemaire V, Oliet SH, Piazza PV, Abrous DN. 2007. Spatial learning depends on both the addition and removal of new hippocampal neurons. PLoS Biol 5:e214.

Ekdahl CT, Kokaia Z, Lindvall O. 2009. Brain inflammation and adult neurogenesis: The dual role of microglia. Neuroscience 158:1021-1029.

Etchamendy N, Desmedt A, Cortes-Torrea C, Marighetto A, Jaffard R. 2003. Hippocampal lesions and discrimination performance of mice in the radial maze: Sparing or impairment depending on the representational demands of the task. Hippocampus 13:197-211.

Etchamendy N, Konishi K, Pike GB, Marighetto A, Bohbot VD. 2012. Evidence for a virtual human analog of a rodent relational memory task: A study of aging and fMRI in young adults. Hippocampus 22:869-880.

Farr SA, Yamada KA, Butterfield DA, Abdul HM, Xu L, Miller NE, Banks WA, Morley JE. 2008. Obesity and Hypertriglyceridemia Produce Cognitive Impairment. Endocrinology 149;5:2628-2636.

Greenwood CE, Winocur G. 2005. High-fat diets, insulin resistance and declining cognitive function. Neurobiol Aging 26 (Suppl 1): 42-45.

He J, Crews FT. 2007. Neurogenesis decreases during brain maturation from adolescence to adulthood. Pharmacol Biochem Behav 86:327-333.

Hwang IK, Kim IY, Kim DW, Yoo KY, Kim YN, Yi SS, Won MH, Lee IS, Yoon YS, Seong JK. 2008. Strain-specific differences in cell proliferation and differentiation in the dentate gyrus of C57BL/6N and $\mathrm{C} 3 \mathrm{H} / \mathrm{HeN}$ mice fed a high fat diet. Brain Res 1241:1-6.

Hwang LL, Wang CH, Li TL, Chang SD, Lin LC, Chen CP, Chen CT, Liang KC, Ho IK, Yang WS, Chiou LC. 2010. Sex differences in High-fat diet-induced obesity, metabolic alterations and learning and synaptic plasticity deficits in mice. Obesity 18:463-469.

Joels M. 2007. Role of corticosteroid hormones in the dentate gyrus. Prog Brain Res 163:355-370.

Kanoski SE, Davidson TL. 2010. Different patterns of memory impairments accompany short- and longer-term maintenance on a high-energy diet. J Exp Psychol Anim Behav Process 36:313-319.

Kanoski SE, Davidson TL. 2011. Western diet consumption and cognitive impairment: Links to hippocampal dysfunction and obesity. Physiol Behav 103:59-68.

Koehl M, Abrous DN. 2011. A new chapter in the field of memory: Adult hippocampal neurogenesis. Eur J Neurosci 33:1101-1114.

Li Y, Dai Q, Jackson JC, Zhang J. 2008. Overweight is associated with decreased cognitive functioning among school-age children and adolescents. Obesity (Silver Spring) 16:1809-1815.

Lindqvist A, Mohapel P, Bouter B, Frielingsdorf H, Pizzo D, Brundin P, Erlanson-Albertsson C. 2006. High-fat diet impairs hippocampal neurogenesis in male rats. Eur J Neurol 13:1385-1388.

Lupien SJ, McEwen BS, Gunnar MR, Heim C. 2009. Effects of stress throughout the lifespan on the brain, behaviour and cognition. Nat Rev Neurosci 10:434-445.

Marighetto A, Etchamendy N, Touzani K, Torrea CC, Yee BK, Rawlins JN, Jaffard R. 1999. Knowing which and knowing what: A potential mouse model for age-related human declarative memory decline. Eur J Neurosci 11:3312-3322.

McNeilly AD, Williamson R, Sutherland C, Balfour DJ, Stewart CA. 2011. High fat feeding promotes simultaneous decline in insulin sensitivity and cognitive performance in a delayed matching and non-matching to position task. Behav Brain Res 217:134-141.

Mielke JG, Nicolitch K, Avellaneda V, Earlam K, Ahuja T, Mealing G, Messier C. 2006. Longitudinal study of the effects of a high-fat diet on glucose regulation, hippocampal function, and cerebral insulin sensitivity in C57BL/6 mice. Behav Brain Res 175:374-382.

Mingaud F, Le Moine C, Etchamendy N, Mormede C, Jaffard R, Marighetto A. 2007. The hippocampus plays a critical role at encoding discontiguous events for subsequent declarative memory expression in mice. Hippocampus 17:264-270.

Molteni R, Barnard RJ, Ying Z, Roberts CK, Gomez-Pinilla F. 2002. A high-fat, refined sugar diet reduces hippocampal brain-derived 
neurotrophic factor, neuronal plasticity, and learning. Neuroscience 112:803-814.

Murray AJ, Knight NS, Cochlin LE, McAleese S, Deacon RM, Rawlins JN, Clarke K. 2009. Deterioration of physical performance and cognitive function in rats with short-term high-fat feeding. FASEB J 23:4353-4360.

Nilsson LG, Nilsson E. 2009. Overweight and cognition. Scand J Psychol 50:660-667.

Park HR, Park M, Choi J, Park KY, Chung HY, Lee J. 2010. A highfat diet impairs neurogenesis: Involvement of lipid peroxidation and brain-derived neurotrophic factor. Neurosci Lett 482:235-239.

Pistell PJ, Morrison CD, Gupta S, Knight AG, Keller JN, Ingram DK, Bruce-Keller AJ. 2010. Cognitive impairment following high fat diet consumption is associated with brain inflammation. J Neuroimmunol 219:25-32.

Privitera GJ, Zavala AR, Sanabria F, Sotak KL. 2011. High fat diet intake during pre and periadolescence impairs learning of a conditioned place preference in adulthood. Behav Brain Funct 7:21.

Richard EM, Helbling JC, Tridon C, Desmedt A, Minni AM, Cador M, Pourtau L, Konsman JP, Mormede P, Moisan MP. 2010. Plasma transcortin influences endocrine and behavioral stress responses in mice. Endocrinology 151:649-659.

Rivera P, Romero-Zerbo Y, Pavon FJ, Serrano A, Lopez-Avalos MD, Cifuentes M, Grondona JM, Bermudez-Silva FJ, Fernandez-Llebrez P, de Fonseca FR, Suárez J, Pérez-Martín M. 2011. Obesity-dependent cannabinoid modulation of proliferation in adult neurogenic regions. Eur J Neurosci 33:1577-1586.

Spear LP. 2000. The adolescent brain and age-related behavioral manifestations. Neurosci Biobehav Rev 24:417-463.

Stranahan AM, Norman ED, Lee K, Cutler RG, Telljohann RS, Egan JM, Mattson MP. 2008. Diet-induced insulin resistance impairs hippocampal synaptic plasticity and cognition in middle-aged rats. Hippocampus 18:1085-1088.

Touzani K, Marighetto A, Jaffard R. 2003. Fos imaging reveals ageingrelated changes in hippocampal response to radial maze discrimination testing in mice. Eur J Neurosci 17:628-640.

Tronel S, Fabre A, Charrier V, Oliet SH, Gage FH, Abrous DN. 2010. Spatial learning sculpts the dendritic arbor of adult-born hippocampal neurons. Proc Natl Acad Sci USA 107:7963-7968.

Valladolid-Acebes I, Stucchi P, Cano V, Fernandez-Alfonso MS, Merino B, Gil-Ortega M, Fole A, Morales L, Ruiz-Gayo M, Del Olmo N. 2011. High-fat diets impair spatial learning in the radial-arm maze in mice. Neurobiol Learn Mem 95:80-85.

White CL, Pistell PJ, Purpera MN, Gupta S, Fernandez-Kim SO, Hise TL, Keller JN, Ingram DK, Morrison CD, Bruce-Keller AJ. 2009. Effects of high fat diet on Morris maze performance, oxidative stress, and inflammation in rats: Contributions of maternal diet. Neurobiol Dis 35:3-13. 